\title{
The LHCb VErtex LOcator and Silicon Tracker Operation Performance Run II
}

\author{
Emma Buchanan*t \\ University of Bristol \\ E-mail: emma.buchanan@bristol .ac.uk
}

The LHCb tracking system includes silicon strip detectors, which are used for the Vertex Locator (VELO) and the Silicon Tracker (ST). These contribute to precise reconstruction of primary and secondary vertices and momentum information for charged particles. Since the beginning of Run I the LHCb experiment has collected more than $7 \mathrm{fb}^{-1}$ of integrated luminosity. The increasing exposure to radiation requires continuous monitoring and modifications to running conditions to maintain a good physics performance. The silicon strip detectors of the LHCb tracking system will be presented with the results of Current-Voltage (IV) and Charge Collection Efficiency (CCE) scan, performed during Run II.

The 26th International Workshop on Vertex Detectors

10-15 September, 2017

Las Caldas, Asturias, Spain

\footnotetext{
${ }^{*}$ Speaker.

On behalf of the LHCb VELO and ST groups
} 


\section{Introduction}

The Large Hadron Collider Beauty (LHCb) Experiment is a single arm forward spectrometer [1], optimised for the study of particles containing $b$ and $c$ quarks originating from $p-p$ collisions at the LHC. The main physics goals are to search for indirect evidence of new physics in CP violation and rare decays of heavy flavour hadrons. The experiment also acts as a general purpose detector in the forward region, with $\mathrm{p}-\mathrm{Pb}$ collisions being studied [2] and the detector taking data as a fixed target experiment [3]. The LHCb tracking system consist of a VErtex LOcator (VELO) [4] detector that surrounds the interaction region reconstructing the primary and secondary decay vertices and a Silicon Tracker (ST), measuring the momenta of charged particles, both using silicon strip detectors. The ST consists of two sub-detectors, the Tracker Turicensis (TT) placed upstream from the magnet and the Inner Tracker (IT) forming the inner part of the tracking stations downstream from the magnet. Since the beginning of the LHC, LHCb has recorded an integrated luminosity of $\sim 7 \mathrm{fb}^{-1}$. The increasing exposure to a high radiation environment has damaging effects on the detectors, therefore the running conditions need to be monitored and adjusted to achieve the best performance. This paper will briefly describe each of the tracking sub-detectors and present the results of Current-Voltage scans and Charge Collection Efficiency (CCE) scans frequently performed to monitor the radiation effects on the detector.

\section{Tracking Sub-Detectors}

\subsection{Vertex Locator}

The VELO consist of 42 semi-circular modules, where each module has a $\mathrm{R}$ and $\Phi$ measuring silicon strip sensor, mounted on either side of a thermally conductive plate that is cooled using evaporative $\mathrm{CO}_{2}$, figure 1(left). The sensors are n-on-n type with a thickness of $300 \mu \mathrm{m}$ and varying pitch, with the exception of one module which is n-on-p. The $\mathrm{R}$ sensor has four $45^{\circ}$ quadrants with the pitch ranging from $40-101.6 \mu \mathrm{m}$ and the $\Phi$ sensor has two regions, with the pitch varying from $35.5-96.6 \mu \mathrm{m}$. The smallest pitch is nearest the beam line, where the occupancy is highest and the best single point resolutions and two-track separation is required. The strips are readout using custom made analogue ASICs placed in the periphery. The VELO is in two retractable halves inside the LHC vacuum, figure 1(right), separated by two $300 \mu \mathrm{m} \mathrm{Al} \mathrm{RF} \mathrm{foils,} \mathrm{figure} \mathrm{1(centre),}$ allowing a secondary VELO vacuum. During unstable beam, the VELO is retracted. During stable beams, the VELO is closed, allowing the first active strip to be $8.1 \mathrm{~mm}$ from the beam.

\subsection{Silicon Tracker}

The ST consists of two sub-detectors [5], both using single sided silicon strip detector layers mounted on a common cooling plate. The TT, upstream of the magnet, has four planar detector layers, where each layer is tilted at a stereo angle of $0^{\circ},+5^{\circ},-5^{\circ}$ and $0^{\circ}$ respectively, figure 2(left). The sensors are $500 \mu \mathrm{m}$ thick p-on-n with a pitch of $183 \mu \mathrm{m}$ and are bonded together into shared readout sectors in groups of 1,2,3 or 4 depending on their proximity to the beam line. This leads to readout strip lengths up to $37 \mathrm{~cm}$. The readout ASICs are in the periphery outside of the acceptance of LHCb. 

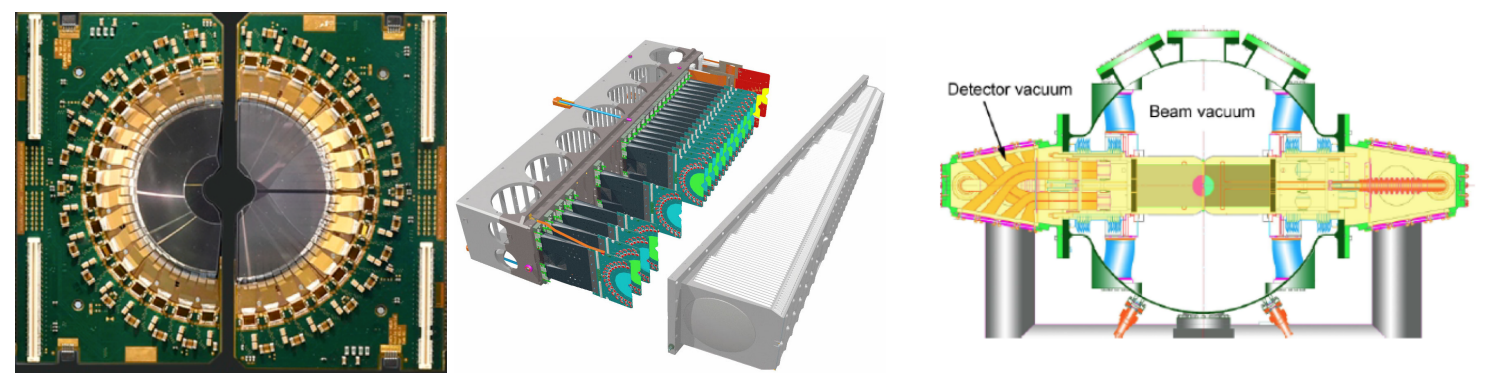

Figure 1: (left) Photograph of two prototype modules, showing one $\Phi \&$ one R measuring sensor with readout ASICs in the periphery. (centre) Illustration of one half of the VELO with corresponding RF foil. (right) Illustration of the full VELO inside the LHC Vacuum.

The IT forms part of the tracking system downstream of the magnet, in the region closest to the beam pipe, figure 2(right), a separate detector, the Outer Tracker (OT) covers the outer regions [6]. There are three tracking stations, where each station consist of 4 layers, tilted in the same way as TT. The sensors are p-on-n with a pitch of $198 \mu \mathrm{m}$, where the thickness depends on the location. The horizontal nature of the magnetic field, means that there needs to be a wider area coverage of silicon sensors on the left and right of the beam pipe. Two sensors are bound together with a readout length of $22 \mathrm{~cm}$, requiring a silicon sensor thickness of $410 \mu \mathrm{m}$ to achieve a sufficient Signal to Noise ratio $(\mathrm{S} / \mathrm{N})$. Above and below the beam pipe, only requires a series of singles sensors, resulting in a readout length of only $11 \mathrm{~cm}$ and allowing a thinner sensor of $230 \mu \mathrm{m}$.
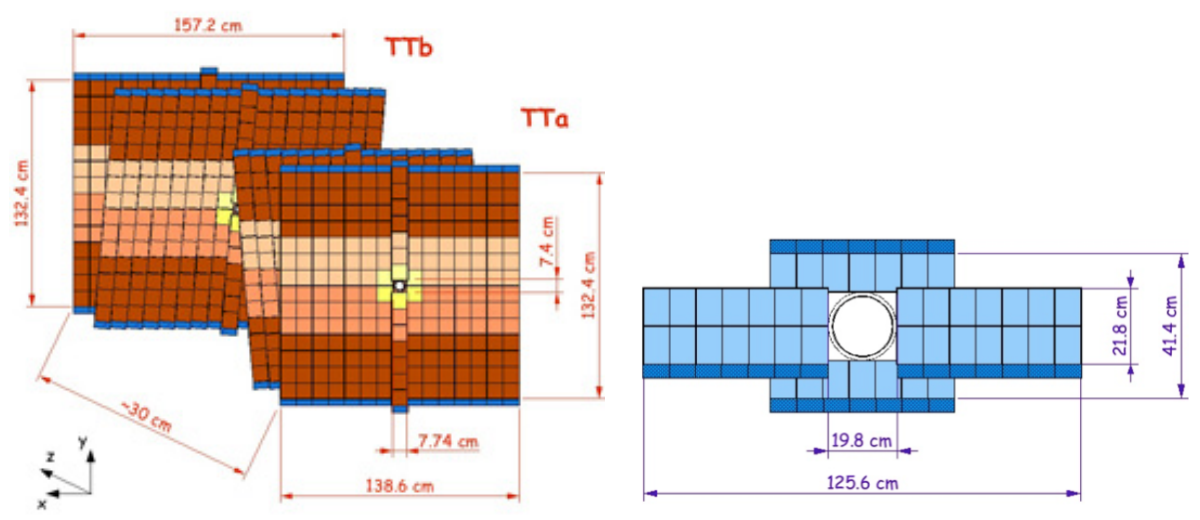

Figure 2: (left) Illustration of TT, where the group readout sectors are highlighted by the different colour shading and the readout is in blue. (right) Illustration of IT, with a greater sensor coverage, left and right of the beampipe.

\subsection{Expected Fluences}

LHCb is designed to run at a lower luminosity $\left(2 \times 10^{33} \mathrm{~cm}^{-2} \mathrm{~s}^{-1}\right)$ than ATLAS and CMS, to avoid excess pileup of p-p interactions. The forward geometry of LHCb means that the radiation environment is highly non uniform. The expected fluences have been estimated using simulations [7]. For the VELO, at $14 \mathrm{TeV}$, the maximum fluence at the inner most irradiated area of the sensors, is of the order of $6 \times 10^{13} 1 \mathrm{MeV} \mathrm{n}$ eq $/ \mathrm{cm}^{2}$ per $\mathrm{fb}^{-1}$ [8], where the fluence varies as $1 / \mathrm{r}^{2}$, figure 3 (left). 
The $\mathrm{z}$ position of the sensor relative to the interaction region, also significantly affects the fluence. The TT expects a maximum fluence of $\sim 10^{13} 1 \mathrm{MeV} \mathrm{n} \mathrm{eq}_{\mathrm{eq}} / \mathrm{cm}^{2}$ per $\mathrm{fb}^{-1}$ [9], where there is three orders of magnitude difference across the sensor, figure 3(centre). The IT, expects a peak fluence of $\sim 10^{12} 1 \mathrm{MeV} \mathrm{n}$ eq $/ \mathrm{cm}^{2}$ per $\mathrm{fb}^{-1}$, however the profile differs from the upstream trackers due to the presence of the magnetic field, deflecting charged particles in the horizontal plane, figure 3(right).
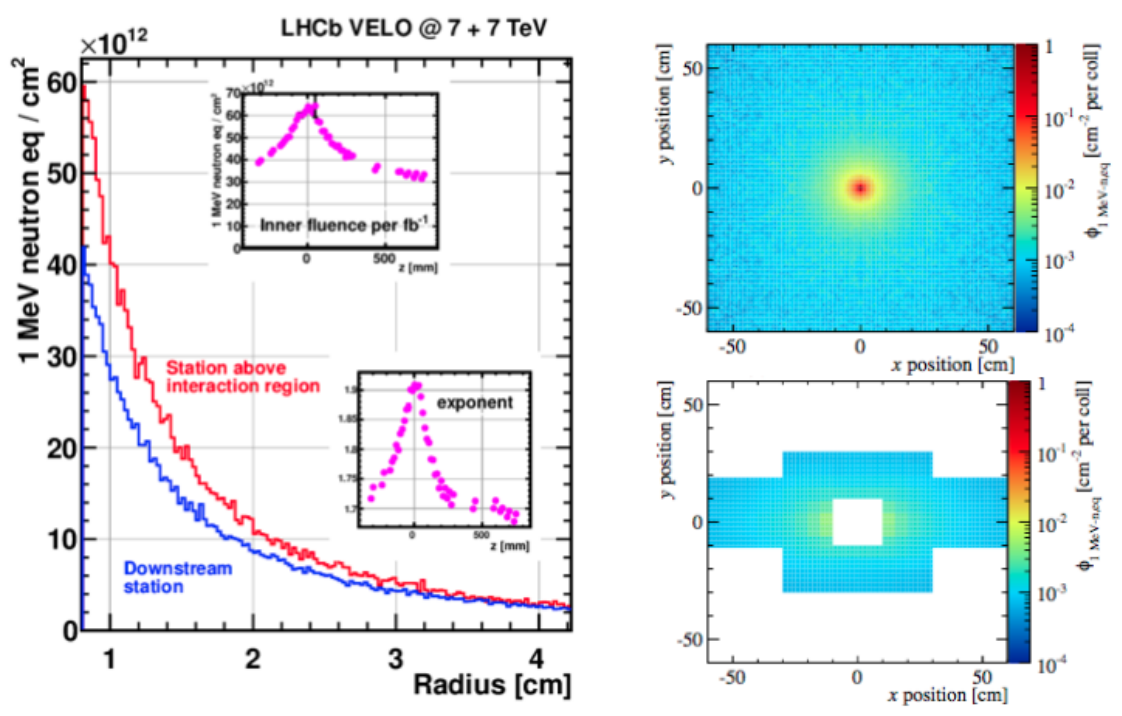

Figure 3: The fluence per $\mathrm{fb}^{-1}$ versus radius for two VELO sensors, one above the interaction region (red) and one downstream (blue), simulated for collisions at a $7 \mathrm{TeV}$ centre-of-mass energy. TT (top) and IT (bottom) are presented as per collision at a centre-of-mass energy of $4 \mathrm{TeV}$, demonstrating the fluence profile, before and after the LHCb magnet.

\subsection{Leakage Currents}

Particle irradiation leads to damage in both the surface and bulk of silicon sensors [10]. The main cause of bulk radiation damage, is the displacements of atoms in the silicon from their lattice positions, leading to an increase in the leakage current and changing the effective doping concentrations. The leakage current varies linearly with fluence and therefore is a good quantity to measure the radiation damage. Consequently, weekly IV scans were performed for all of the LHCb tracking detectors during periods when there was no beam.

\subsubsection{VELO}

The measured leakage currents over time for the VELO are shown in figure 4. Each of the purple trends represents a VELO sensor, where the variations in current are partly due to the different sensor positions relative to the interaction point, but are dominated by temperature variations between sensors. The mean measured current (green) agrees well with the predictions from simulation (pink). The areas of near constant currents are measurements taken during Long Shut Downs and Technical Stops, where in some instances annealing can be observed when there are drops in currents after warm up periods. The delivered luminosity and average sensor temperatures are presented at the top of the figure, where periods of shutdown can be identified. The VELO sensors are 
maintained at $-30{ }^{\circ} \mathrm{C}$ when not data taking and at $-7{ }^{\circ} \mathrm{C}$ when taking data. During shutdowns the temperature is maintained between $-11^{\circ} \mathrm{C} \&-38^{\circ} \mathrm{C}$ for vacuum (neon) conditions.

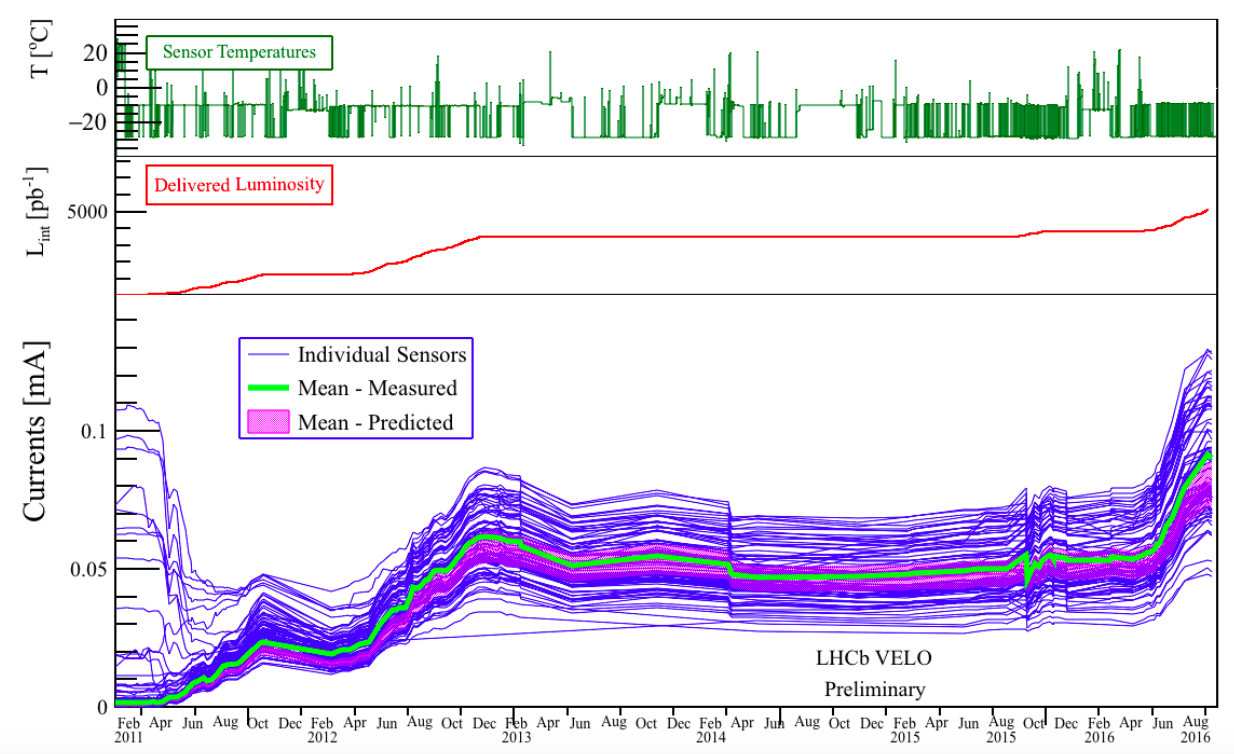

Figure 4: The leakage currents for the VELO as a function of time. Above, the delivered luminosity and the average temperature variations.

The variations in leakage current due to the sensor positions in $\mathrm{z}$, relative to the interaction point can be seen when the temperatures are normalised. Figure 5, shows the VELO leakage currents depending on the $\mathrm{z}$ position of the module. The currents have been taken at $200 \mathrm{~V}$ and the temperatures were normalised to $-10{ }^{\circ} \mathrm{C}$, clearly displaying the differences in currents due to the proximity of the interaction region of the modules. The highest currents are seen for the modules closest to the interaction region at $\mathrm{z}=0$. The different data point colours represent different measurement dates. A significant difference can be seen between, 2015 and 2016 winter shutdowns, due to LHCb collecting 5 times more integrated luminosity in 2016 than 2015.

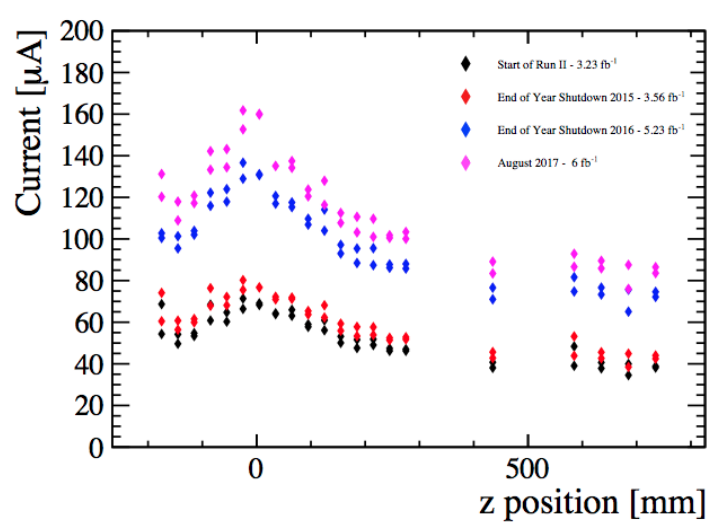

Figure 5: VELO leakage currents depending on the $\mathrm{z}$ position of the module. 


\subsubsection{Silicon Tracker}

The leakage currents are shown for ST in figure 6, TT (left) and IT (right). Both have been normalised to a temperature of $8^{\circ} \mathrm{C}$, but are operated at different bias voltages due to different sensor thicknesses. The trends are very similar to the VELO, with dips in current during shutdowns. For the TT (left) the two colours represent two different detector planes (inner $\pm 5^{\circ}$ ), the sensors closest to the beam line have the highest currents as expected. For the IT (right), the trend colours represent the different sensor locations. Due to the horizontal nature of the magnet, the highest fluence for the IT is on the left (red) \& right (blue) side of the beam pipe, leading to higher currents for these sensors compared to those in the top (green) \& bottom (pink).
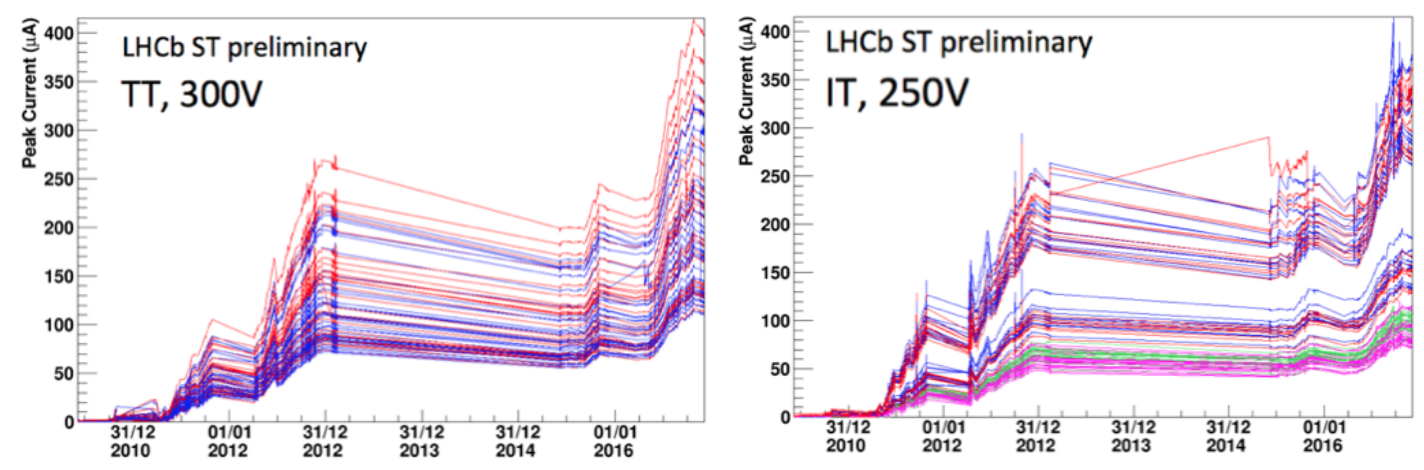

Figure 6: The Leakage currents for the ST, (left) TT, (right) (IT)

\subsection{Charge Collection Efficiency}

The radiation induced change in the doping concentration leads to the silicon becoming more p-type, causing type inversion of the initial n-bulk to p-bulk [11], followed by a steady rise in the bias voltage required to operate the detector. This results in a change in the effective depletion voltage. The depletion voltage can be measured by performing a Charge Collection Efficiency (CCE) scans, taken four times a year during dedicated data taking, typically during periods of low luminosity operation.

\subsubsection{VELO CCE Scans}

For the VELO, every $5^{\text {th }}$ module is removed from the tracking and the bias voltage is varied, while all other modules are run at their nominal voltage. An estimate of the hit position can be determined using reconstructed tracks in the nominal planes. The signal amplitude is the sum of the signals of the three strips closest to hit position. A landau is then fit to the ADC distribution to determine the Most Probable Value (MPV).

Figure 7(left) is an example of the ADC distributions, with Landau fit, for a range of voltages. Figure 7(right) is the MPV as a function of bias voltage, where the sensor has been separated into 5 radial bins. The red line represents the inner most radial bin, closest to the interaction region and therefore has a higher depletion voltage compared to the blue line, the bin that covers the outer most radii and hence the least radiated. The effective depletion voltage (EDV) is defined as the the voltage at which the MPV of a sensor is equal to $80 \%$ of the maximum. 

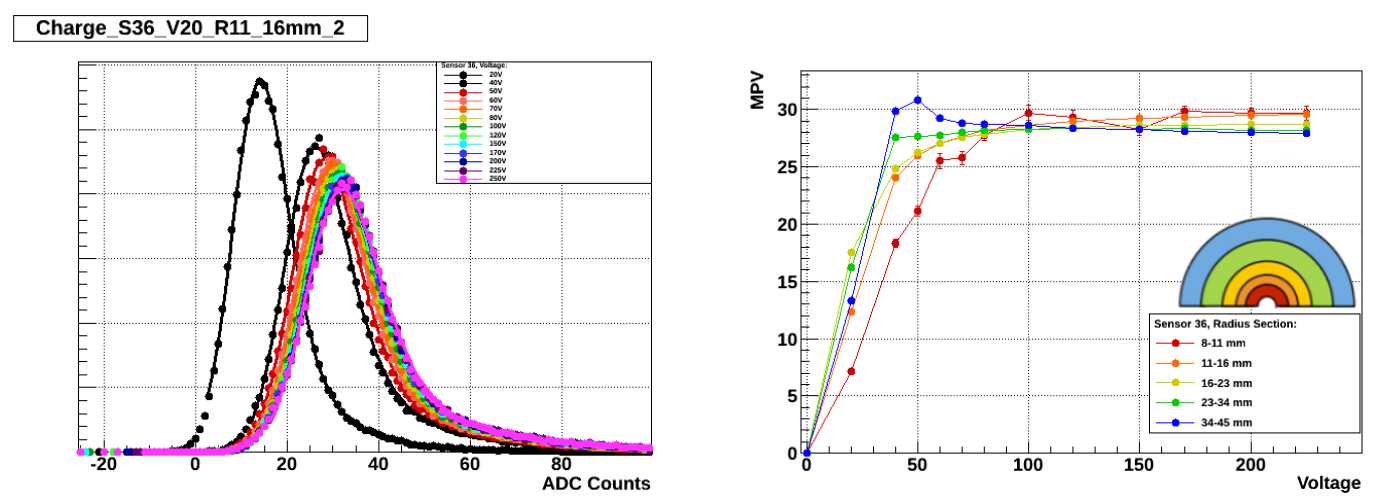

Figure 7: (left) The ADC distributions for a range of bias voltages. (right) The MPV as a function of bias voltage depending on the radial bin. These are an example of the method, using data from 2015.

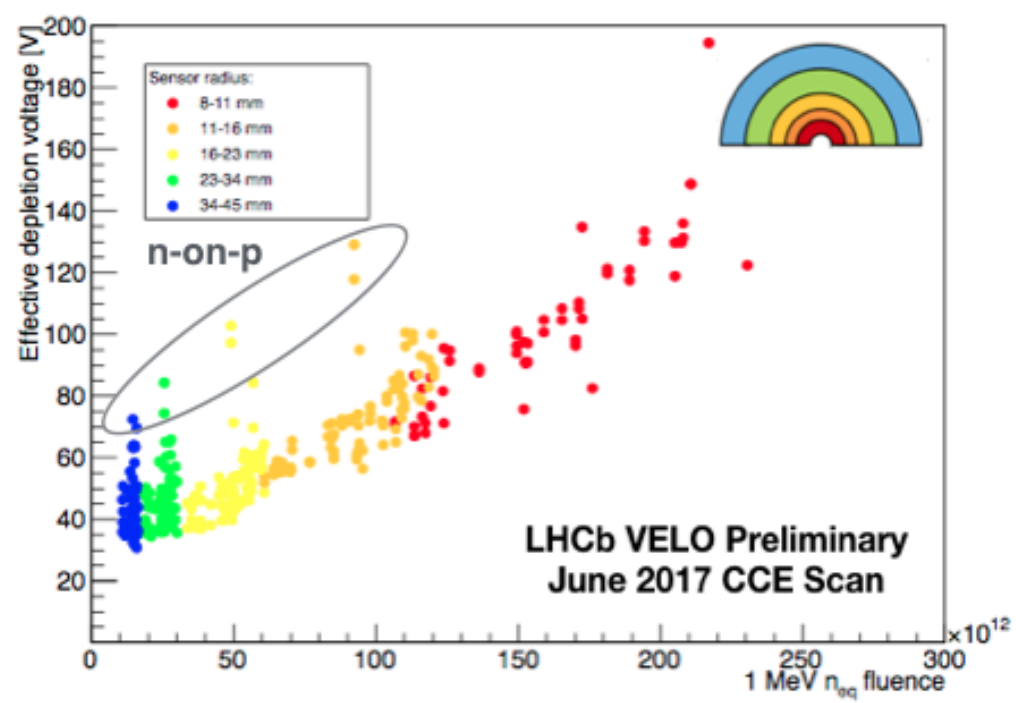

Figure 8: The effective depletion voltage for all VELO sensors, depending on radial bin. The data was collected after a delivered integrated luminosity of $5.7 \mathrm{fb}^{-1}$.

Figure 8 is a plot of the EDV as a function of fluence, for all VELO sensors, depending on radial position. As expected, the inner radial bins have the highest EDV due to increased radiation damage compared to the outer radii. The operational voltage of the VELO was set to $300 \mathrm{~V}$ at the beginning of 2017 to ensure full depletion of the sensors.

\subsubsection{ST CCE Scans}

For the ST, the CCE scan method is similar to the VELO, however only one plane is removed from the tracking. The voltage of the test plane is varied and the MPV is measured using a landau fit to the ADC distribution, figure 9(left). This is repeated for different time shifts to obtain a pulse shape [12], figure 9(centre). The bias voltage influences the charge collection speed, by performing a timing scan it can be assured all charge is collected. The total collected charge is then estimated by integrating the pulse shape, and plotting as a function of bias voltage, figure 9(right). The 
resulting curve is fit with a spline and the depletion voltage is then defined as the bias voltage at which the fit reaches $95 \%$ of the maximum value.
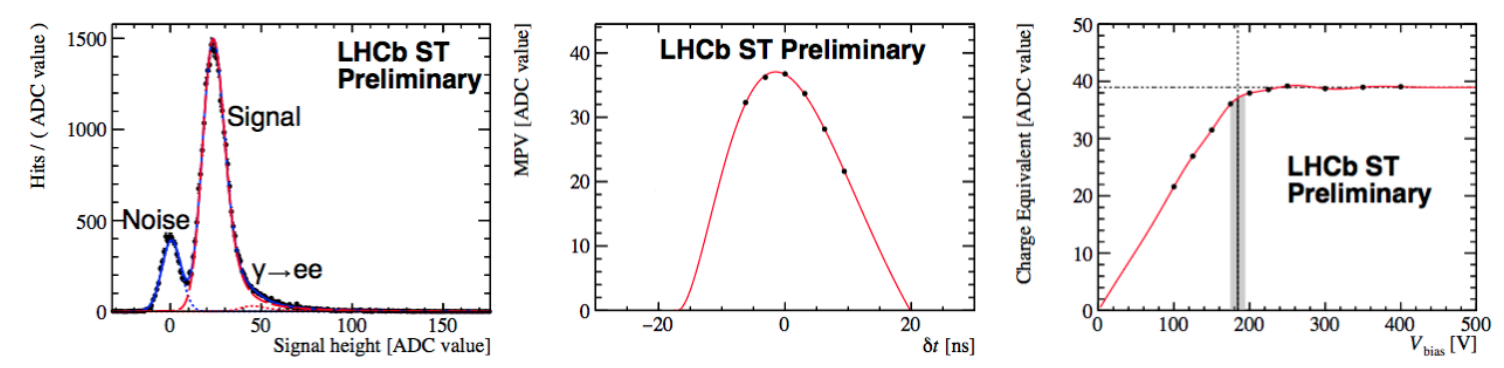

Figure 9: Example for TT, (left) the ADC distribution, (middle) the pulse shape, obtaining the MPV for different time shifts for a set voltage. (right) The total collected charge as a function of bias voltage.

The effective depletion voltage for TT and IT as a function of fluence are shown in figure 10, and are normalised to the depletion voltage before irradiation. The effective depletion voltage for TT decreases with increasing fluence because both detectors sensors have not yet reached type inversion. The dotted lines represent the Hamburg model [10] predictions, showing a good agreement between measurements and predictions.
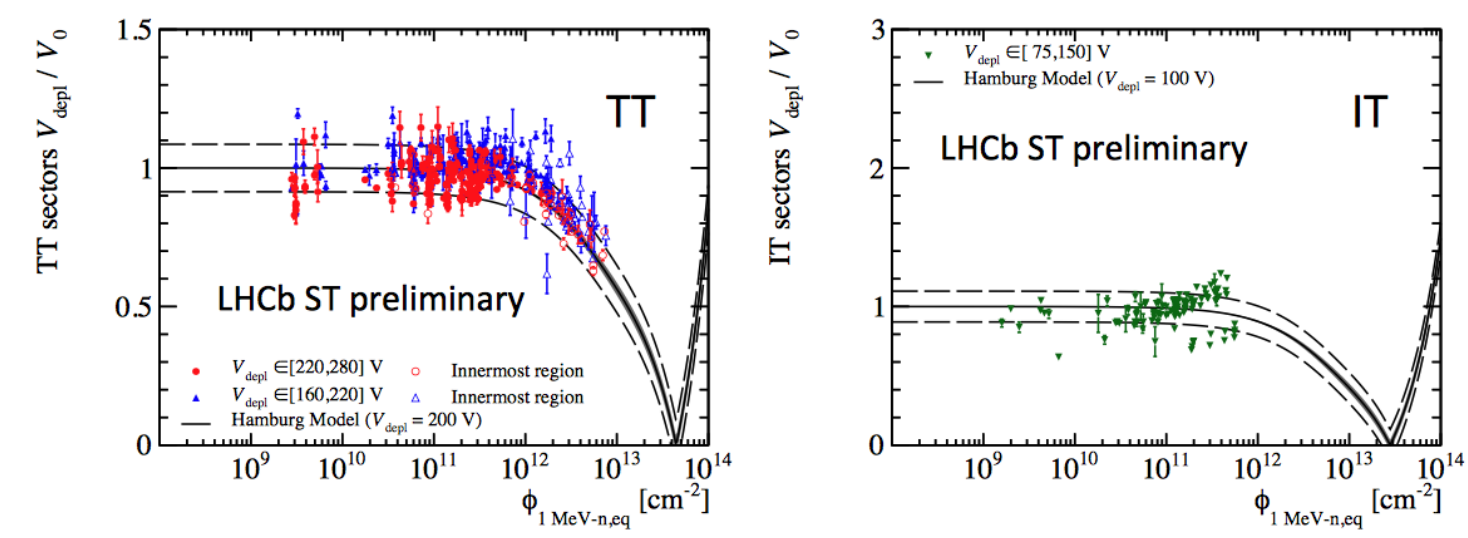

Figure 10: The depletion voltage for (left) TT and (right) IT, normalised to the depletion voltage before irradiation.

\subsubsection{Cluster Finding Efficiency}

Efficient track reconstruction is vital for a good physics performance. As a consequence of radiation damage, clusters can be lost due to charge trapping [10], reducing the cluster finding efficiency (CFE). The method described previously for the CCE scan can also be used to measure the CFE for the VELO, by looking for the presence of a cluster at the track intercept on the test sensor. At the start of Run I, the majority of sensors had a mean efficiency greater than $99 \%$, uniformly distributed across the sensor. The CFE for a $\Phi$ sensor, taken in Run II, is shown in figure 11(left), which illustrates the CFE across the sensor and (right) is the mean CFE as a function of radius for 4 different scans. The efficiency is lower at the innermost radii, closest to the interaction region. 
There is a small dip in efficiency at the boundary between the inner and outer strips. The majority of the $\Phi$ sensor after $5 \mathrm{fb}^{-1}$ is $\sim 98 \%$ efficient.
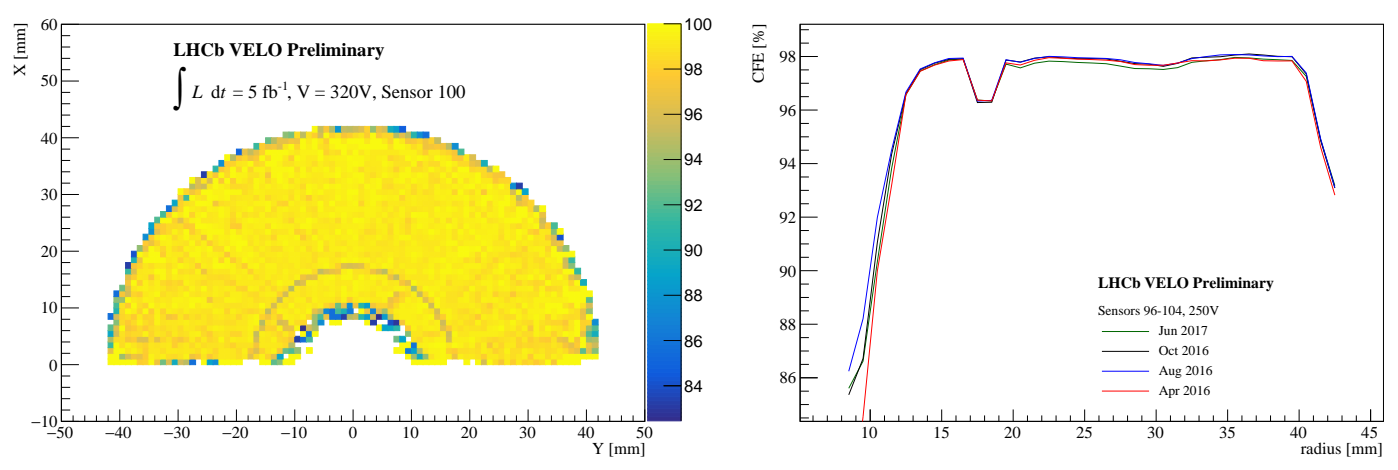

Figure 11: The CFE for a $\Phi$ sensor, (left) full sensor, (right) average CFE as a function of radii for four different scans taken in Run II

The CFE for a R sensor, also taken in Run II, is shown in figure 12. In contrast to the $\Phi$ sensor, the CFE decreases significantly with increasing radii. Similarly to $\Phi$, the efficiency is lower in the inner most radii, however in the lower luminosity regions, the efficiency is less. This effect is a consequence of the routing lines, a second metal layer that carries the signal from the inner strips to the readout in the periphery [8]. A charged particle traversing close to a routing line, induces a charge on the routing line, registering a hit on another strip. In most cases the strip is outside of the search window of associating clusters to a track, resulting in a lower CFE. Since the reduced efficiency is present in the outer radii of $\mathrm{R}$ sensors, far from the interaction region, it has limited effect on the tracking performance. This is not seen in $\Phi$ sensors as the routing lines run parallel to the strips, whereas for R, the routing lines are perpendicular and run over the top of the strips. A more detailed discussion on the double metal layer can be found in Reference [8].
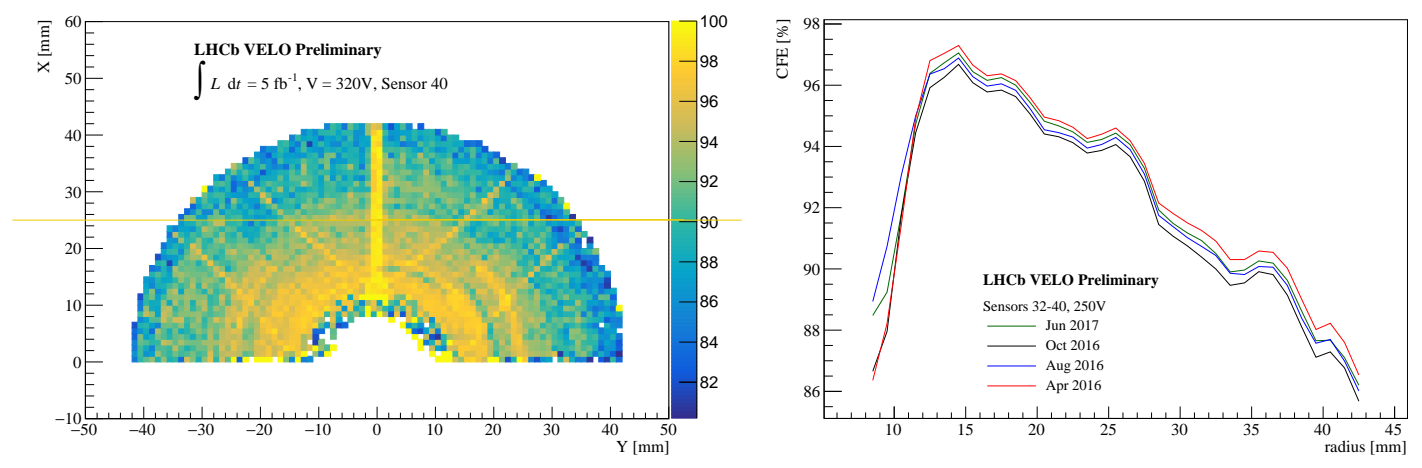

Figure 12: The CFE for a R sensor, (left) full sensor, (right) average CFE as a function of radii for four different scans taken in Run II

\section{Conclusions}

The LHCb tracking detectors are performing well during Run II. The radiation damage is 
monitored by measuring the leakage currents and the effective depletion voltages. All tracking sub-detectors have increased leakage currents as expected, with occasional annealing during extended periods of no beam. Significant radiation damage to the VELO has lead to type inversion of its sensors, causing an increase in depletion voltages. The depletion voltages for the ST are gradually decreasing as they have not yet reached type inversion and are following predictions well. The VELO has reduced CFE efficiency in the outer radii of R-sensors due to the double metal layer, however there is minimal effect on the tracking performance. The radiation damage is not expected to result in problems for the LHCb tracking systems before the end of Run II, after which the detectors will be upgraded during Long Shut Down II (2019) to allow operations at higher luminosities.

\section{References}

[1] LHCb : Technical Proposal. Tech. Proposal. CERN, Geneva, 1998.

[2] Prompt and nonprompt $\mathrm{J} / \Psi$ production and nuclear modification in $p P b$ collisions at $\mathrm{sNN}=8.16 \mathrm{TeV}$, Physics Letters B (2017) .

[3] LHCB collaboration, E. Maurice, Fixed-target physics at LHCb, in 5th Large Hadron Collider Physics Conference (LHCP 2017) Shanghai, China, May 15-20, 2017, 2017, 1708.05184 , http://inspirehep.net/record/1616496/files/arXiv:1708.05184.pdf.

[4] R. Aaij et al., Performance of the LHCb Vertex Locator, JINST 9 (2014) 09007, [1 405 . 780 8].

[5] M. Tobin, The lhcb silicon tracker, Nuclear Instruments and Methods in Physics Research Section A: Accelerators, Spectrometers, Detectors and Associated Equipment 831 (2016) $174-180$.

[6] Performance of the lhcb outer tracker, Journal of Instrumentation 9 (2014) P01002.

[7] R. Wunstorf, M. Benkert, N. Claussen, N. Croitoru, E. Fretwurst, G. Lindstrom et al., Results on radiation hardness of silicon detectors up to neutron fluences of $1015 \mathrm{n} / \mathrm{cm} 2$, Nuclear Instruments and Methods in Physics Research Section A: Accelerators, Spectrometers, Detectors and Associated Equipment 315 (1992) 149 - 155.

[8] A. Affolder et al., Radiation damage in the LHCb Vertex Locator, JINST 8 (2013) P08002, [1302.5259].

[9] C. Elsasser, The Rare Decays $\mathrm{B}_{(\mathrm{s})}^{0} \rightarrow \mu^{+} \mu^{-}$and $\mathrm{Z}$ Boson Production at LHCb and Radiation Damage in its Silicon Tracker, Feb, 2015.

[10] M. Moll, Radiation damage in silicon particle detectors: Microscopic defects and macroscopic properties, Ph.D. thesis, Hamburg U., 1999.

[11] R. Cannara, B. Dezillie, T. Dubbs, J. Hancock, W. Kroeger, Z. Li et al., Depletion voltage and charge collection for highly irradiated silicon microstrip detectors with various initial resistivities, IEEE Transactions on Nuclear Science 46 (Dec, 1999) 1964-1968.

[12] C. Elsasser, The lhcb silicon tracker performance \& radiation damage, Nuclear Instruments and Methods in Physics Research Section A: Accelerators, Spectrometers, Detectors and Associated Equipment 730 (2013) 13 - 17. 